\title{
Falencias y falacias: notas sobre el estudio de las relaciones Norte-Sur
}

El pensamiento la!inoamericano ha tenido una influencia decisivat en el surgimiento de las itlcas y las instituciones que actualmente sirven de base a los esfuerzos encrminados al establecimiento de un Nuevo Orden Económico Internacional (NOEI).

"Tengo muy pocas duclas en que el liderazgo intelectual del Tercer ifundo, en lo que se refiere a los problemas del desarrollo económico, es firmemente ejercido por un sofisticaclo, imaginativo y dedicado grupo de latinoamericanos. Ellos han creado los conceptos relacionados con la OPEP, la indexación, la miniclevaluación ya la desinversión. Ėllos están promoviendo activamente la adopción de códigos internacionales de conducta $y$ un mayor control sobre las corporaciones multinacionales. Cuando las relaciones económicas entre los países desarrollados y los paises en clesarrollo se discuten en los foros inter: nacionales, los representantes latinoamericanos son actores estelares y elocuentes autores de exhaustivos documentos de trabajo, cuidadosamente prejarados en Brasil, México o Venezuela. Los latinoamericanos no solamente son los lideres tcóricos del Tercer Mundo. Ellos han sido pioneros en el establecimiento de nuevos códigos, filosof́as, leyes y regulaciones en sus propios paises, empeñándose asi en llevar a la práctica las icleas de sus intelectuales y tecnócratas. Estos expcrimentos son observados cuidadosamente por otras naciones en desarrolio y pueden proporcionar las bases para la adopción de programas similares en Africa, el Sudeste Asiático y en todo el mundo".

Estas palabras escritas por el señor José de Cubas, presidente honorario del Consejo de las Américas, en la introducción del Informe Anual de esa organización, correspondiente a 1975, constituyen un tsstimonio insospechablemente objetivo acerca de la importancia que ha tenido el pensamiento latinoamericano en el replanteamiento del orden internacional, durante los últimos 25 años. 
Cuando América Latina planteó la necesidad de realizar un examen crítico de las relaciones centro-periferia, principalmente a través de los análisis de don Raúl Prebisch y de sus primeros colaboradores en la Comisión Económica para América Latina (CEPAL) ${ }^{2}$, no había prácticamente nadie que desde una perspectiva no maxista cuestionara el orden internacional enraizado en el clesarrollo y ulterior liquidación de los imperios coloniales y consagrado, hacía pocos años, en los acuerdos de Bretton Woods. Eventualmente, el pensamiento latinoamericano, acuñado en la CEPAL, alcanzó resonancia mundial a través de la UNCTAD, y condujo a un cuestionamiento generalizado del orden establecido.

Sin embargo, paradojalmente, hoy parece darse una situación inversa: Ios planteamientos referentes a la necesidad de un nuevo orden internacional provienen de otras regiones del mundo en vías de desarrollo -o de paises industrializados que tienen como marco de referencia las caracteristicas y problemas de sus antiguas esferas de influencia y que están muy poco familiarizados con la realidad latinoamericana- mientras que en América Latina se hace sentir la falta de un análisis critico y de una respuesta propia frente a esos planteamientos. Si bien el último estudio de la CEPAL contiene percepciones que abren el camino hacia una interpretación diferenciada acerca de la posición de América Latina entre el resto de los países en desárrollo y dentro del sistema internacional en su conjunto", pareciera: que estas percepciones no han motivado aún a aquellos grupos académicos independientes que tanta influencia tuvieron sobre la formación del pensamiento latinoamericano en el pasado, pero cuya contribución podría debilitarse en la medida en que vayan quedando a. la zaga de la realidad.

Mirando más allá de América Latina, cabría abrigar el temor de que incluso planteamientos originados en otras latitudes, como los:anteriormente mencionados, no estuvieran fundados en, una base de análisis adecuada, lo que contribuiría a explicar sus magros resultados. Descle luego, los primeros análisis de las relaciones centroperiferia realizados desde el punto de vista de los paises en desarroflo-o, para ser más precisos, desde una perspectiva latinoamericana- no han alcanzado el status que probablemente merecían en los círculos académicos de los países industrializados; el dia en que esos trabajos pioneros reciban un reconocimiento de la importancia del:Premio Nobel, por ejemplo, las ciencias sociales cle estos países .

\footnotetext{
I Prebiscl, Raúl, Problemas leóricos y prúcticos del crecimiento económico, CEPAL, 1959.

¿CEPAL, El Desarrollo Económico y Social y las Relaciones Externas de América Latina, 1977.
} 
habjáin dado un nuevo y decisivo piso para salir del suntuoso, pero no por ello menos parroquial, enclaustraniento en que se encuentran. Ademas, da la impresión de que los esfuerzos realizados para analizar estas relaciones, tomados en conjunto, han adolecido de algunas limitaciones. En primer lugar, han tendido a adoptar un punto de vista estático, y no dinámico, lo que les ha creado dificultades para reconocer las nuevas situaciones planteadas a los países en desarrollo por la evolución del sistema internacional, en su conjunto, $y$ de sus propias economias, -así como también, aunque tal vez en menor: mediclia, para adverlir las múltiples implicancias del proceso de transnacionalización que ha estado experimentando la economía mundial con especial celericlad durante los últimos 15 años, y para lievar estos anilisis hasta un plano más profundo que el de las relaciones comerciales entre el centro y la periferia. En el segundo lugar, como ya se hia anticipado, ha resultado difícil percibir que, como consecuencia de la creciente interdepenclencia del sistema transnacional, el desarrollo y el subdesarrollo forman parte de un mismo proceso - siendo el subdesarrollo de la periferia causa y efecto, a la vez, del desarrollo de los centros - lo cual ha conducido, por una parte, al prestar poca importancia al comportamiento de los centros para estudiar la evolución y perspectivas de los países periféricos $y$, por la otra, a proponer estralegias que hacen depender sustancialmente el proceso de desarrollo de la adopción unilateral, por parte de estos últimos, de politicas cconómicas "sanas". En tercer lugal, durante largo tiempo se experimentaron mayores dificultades que en cl resto de las ciencias sociales para integrar los factores políticos y económicos en cl análisis de las relaciones internacionales, dificultades que entorpecieron el entendimiento $Y$ la coo. peración entre diplomáticos $y$ tecnócratas $y$ que incluso fueron visibles en la conducción superior de la política exterior de los Estados Unidos durante la última administración republicana, no obstante que en el plano conceptual cstaban en vías de ser superadas mediante el aporte reciente de un grupo de lúciclos analistas de estos pro. blemas ${ }^{3}$.

Lo anterior se ha traducido en un alto grado de polarización en el análisis de las relaciones internacionales entre dos corrientes de pensamiento inspiracias, respectivamente, en una filosofia "librecam-

3 Part una de las primeras y más lúcidas exposiciones de este punto de vista, ver C. Fred Bergsten, The Future of the International Economic Orders: An Agenda for Research, 1973. Ver tambicin C. Fred Bergsten y Lawrence Krause (editores). JVorld Politics and Internalional Économics, 1975; Robert Gilpino, "The Politics of Transnationa! Economic Relations", en Rober' O. Keohane y Joseph S. Nye, Transnational Relations and World Politics, 1971 y Richard Cooper: "Trade Policy is Foreign Policy", en Foreign. Policy, No 9, 1972.73. 
bista" y en una filosofia "intervencionista" frente al actual funcionamiento $y$ a la evolución futura de la cconomia internacional, impidiendo o retrasando la a parición de interpretaciones más desagregadas o matizadas, tema al cual se hará referencia en la última parte de estas notas.

En estas notas se procura señalar en. forma tentativa algunas falencias y falacias que se observan en cl anúlisis de las relaciones Norte-Sur, sin circunscribirnos a la labor realizada en tal sentido descle América Latina, pero con especial referencia a la peculiar -y de los países en desarrollo- y al aporte fundamental que el pensamiento latinoamericano deberia efectuar una ve\% más para la comprensión de estos problemas.

\author{
PERSPECTIVAS DEL TERCER MUNDO \\ EN EL ACTUAL CONTEXTO INTERNAGIONAL
}

Los esfuerzos encaminados a la formulación y establecimiento de un Nuevo Orden Económico Internacional se ven debilitados, a nuestro juicio, por el hecho de estar basados en tres lalacias principales.

Antes de relerimos a ellas, es preciso reconocer que la actual coyuntura resulta particularmente oportuna para la realización. de esfuerzos encaminados a la reforma del sistema internacional, de acuerdo con lineamientos mis favorables para los intereses del Tercer Mundo. En efecto, a las legítimas aspiraciones revisionistas de estos países ha venido a añadirse la gradual toma de conciencia de que vivimos en un mundo cada vez más interdcpendiente por parte de las naciones industrializadas.

Las relaciones internacionales, durante el decenio de 1970 , se han caracterizado por la emergencia de una relación de interdependencia entre todas las naciones del mundo en una escala que no habría podido ser imaginada hace 25 años atrís. Es evidente que problemas tales como la "estagflación" que han experimentado recientemente

4 I'ara una exposición clasica del primer pumo de vista, ver Harry Johnson, Economic Policies \%oward Less Developed Conntries, $1967, y^{+}$.'The Link that Chains", en Foreign Policy, No 8, 1972. Lit antiguo embajactor en lis India $y$ en las Naciones Uniclas, D. Patrik Noy'rihan, por su parte, sugirió en un artículo titulain "The U.S. in Opposition", publicado en Commentary en marzo de 1974 , que la posicjón adoptada por los patises del "rercer Mando acusaba una fuerte influencia del movinicnto laborista ingles y ted pensamiento de la Siciedad Faljiana. Esta generalización indelida demestra, precisamente, hasta qué punto Asia y Africa han pasido a conslituirse en los voceros del NoEI, pues el pensimiento fabjatno jamás ejerció tinfuencia en Latinoamérica. 
los países industrializados, el alunento del precio de los conbustibles, alimentos $y^{\prime}$ fertilizantes, y las tendencias hacia la cscase\% de recursos naturales y el cieterioro ecológico, ha produciclo un profundo impacto sobre el sistema internacional, impacto al cual no escapa ningún país ni grupo de paises. lis por eso que muchos observatores han hegado a la conclusión de que estos problemas globales sólo podrán ser resueltos mediante la acción concertada cle todas las nacioncs clel mundo. De hecho, muchas de estas cuesliones, tales como lat re. forma del sistema monetario internacional, la aclopción cle un régimen para la explotación de los recursos marinos, la energía y la ali. mentación, están siendo abordados, a través de foros auténlicamente. muncliales. Si bien muchos cle estos problemas requerirán consultas mis intensas entre los paises industrializaclos clel Norte, necesidad que durante la anterior administmaión norteamericana se tradujo en la creación de la Comisión Trilateral, llamada a constituir una "comunidad de las naciones desarrolladas", de acuerdo con las palabras de su primer clirector, el profesor Sbigniew Brzezinski, no hay duda de que en muchos de ellos se requeriá la colaboración. de los paises del Sur.

La impresión de que el momento es propicio para aratuar hacia cl establecimiento cle un Nucro Orden Éconómico Internacional se fundat en lat aprecinción cie que los paises del Tercer Mundo tendrian mucho que gatuar en la actual coyuntura internacional en la medida en que conserven su uniclad de acción y de propósitos. ¿En qué se basa esta apreciación $y$ hasta quć punto es factible la solidaridad del Tercer Mundo?

Líl tenclencia a la clispersión del poder económico mundial, como consecuencia de la aparicion. de nuevos centros de alto clesarrollo industrial, tecnológico y financiero, además de los Estados Unidos, podría abrir nuevats oportunidades part diversilicar los mercados y las fuentes de abastecimiento de bienes de capitill, de tecnologia y de financiamiento de que actualmente disponen los paises en destrrollo, particularmente si éstos procuran aprovechar estas oporLunidades al thués de una acción concertacla. Al mismo tiempo, el ensanchamiento del margen de seguriclat de las grandes potencias, derivalo de la declinación cle lá guerra friat, y la rclevancia que hán pasaclo al adịuirir los intereses económicos en las negociaciones internacionates, han determinato que los objetivos relacionalos con la esfera de la seguridad se consideren cada vez más vinculados con factores de orden económico. Estos íltimos, por consiguiente, adquieren calda vet, más peso et la formulación de la politica exterior. Illo, a su vez, podriat contribuir a mejorar la participación de los paises en desarrollo dentro del sistema internacional, especialmente si ac- 
túan de consuno, toda vez que $\mathrm{cl}$ poder económico estii relativamente menos concentrado que el poderio político y militar.

Al mismo tiempo, los paises industrializados continúan asignalndo una aita prioridad a las políticas antiinflacionarias. Dentro de este contexto, el aprovechamiento de las ventajas comparativas que poseen algunos paises del Tercer Mundo para producir determinatlas manufacturas y una búsqueda más racional de la división internacional del trabajo de acuerdo con lineamientos similares a los que tradicionalmente han preconizado los países industrializados, conjuntamente con el comportamiento ordenado de los precios cle los combustibles y las materias primas, han pasado a constituir un elemento esencial para la evolución de las economias de los paises del Norte.

Las elevadas tasas de crecimiento que han experimentado las economías industrializadas durante los últimos decenios han ido acompañadas, en los años recientes, por una preocupación generalizada acerca del posible agotamiento de lit capacidad de respuesta del ecosistema a los requerimientos planteados por esos mismos procesos, particularmente en lo que se refiere al alsastecimiento cle recursos naturales, asignando especial importancia a las consideraciones relativas a la "seguridad económica". Por consiguiente, el acceso a las fuentes cle suministro de materias primas, un sector cle especial interés para los paises del Terccr- Mundo, ha pasado a constituir otra preocupación fundamental para los paises industrializados.

Es cierto que estas oportunidades se ven parcialmentc erosionadas por el nuevo mercantilismo que parece haberse entronizaclo en las relaciones internacionales como consecuencia de las presiones que se ejercen en los países capitalistas del Norte para garantizar el pleno empleo y el bienestar de sus ciudaclanos, amenazados por las tendencias recesivas e inflacionarias anteriomente mencionadas. ${ }^{\mathrm{T}}$

Con todo, y recapitulando, si se considera que los paises de la OECD están protagonizando un proceso de dispersión del poder econónico mundial, continúan dando mixima prioridad al problema de la inflación y están crecientemente preocupaclos frente al fantasma de la escasez, se llega a la conclusión de que los países del Tercer Mundo tendrian mucho que ganar con una mayor diversificación de sus relaciones económicas cxteruas, con una inserción más eficiente en la divjsión internacional del trabajo y de un mejor aprovechamiento de los beneficios clerivados de la extracción $y^{t}$ trans. formación de sus recursos naturales.

El logro de esas ganancias requeriria una gran sensibilidad para identificar los intereses de ambos grupos de paises y formular solu-

E Ver Erncst IJ. Preeg, Economic Blocks and U.S. Foveign Policy, 1974, Y Harold Aacmgres, "Coming Trade Wars?", en Forcign Policy, NS 1, 1970-71. 
ciones para mutuo beneficio, a través de enfoques más desagregaclos y eventualmente diferentes con respecto a las medidas de carícter general que hasta ahora han configurado la plataforma del Nucvo Orden Económico Internacional.

Queda ahora por examinar la segunda cuestión, acerca de hasta qué punto es efectiva o poclni ser duradera la unidad diel Tercer: Mundo, postulada como condición para obtener las ventajas que podrian ofrecer las oportunidacles en los pinrafos precedentes.

Nuchos observadores ponen en duda la unidad de un conjunto de naciones que presentan tantals diferencias desde el punto de vista de sus tradiciones culturales, sus sistemas politicos y sus niveles de desarrollo económico, alsí como también, clescle el ingulo de su pertenencia a diversos bloques en los cuales se cleja sentir la influencia de las antiguas potencias coloniales del Norte o de los nuevos liderazgos ideológicos que compiten por la hegemonia mundial. Basada cn esa premisa, clurante los últimos años lia diplomacia norteamẹricana ha procurado fragmentar la unidad del Tercer Mundo, como lo revela el trascendido de un cable colocado por el antiguo Secretario de Estado, señor Kissinger, el 22 de noviembre de 1976, que se referia a! "éxito relativo" de los países industriales en sus es[uerzos para intensilicar las presiones ejercidas sobre la OPEP por los demás países en desarrollo, o se desprende de los comentarios formulaclos por el profesor Ullman refiriéndose a la Comisión Trilateral en el sentido de que "una razón esencial para unificar a los paises del Norte es poder negociar más eficazmente con los países del Sur". ${ }^{\circ}$

La unidad del Tercer Mundo, sin embargo, ha desafiado todas Jas predicciones basadas en. los conceptos y estrategias anteriorménte mencionados. Los esfucrzos desplegados clesde 1973 por los palises del Norte - bajo el liderazgo de los Estados Unidos y con la renuen. cia de Francia- para aislar a los países de la OPEP, basaclos en 'la percepción de que "cl petróleo sería la excepción" , se vieron frustrados cuando aquellos estados insisticron en negociar los problemas relativos a la energía conjuntamente con los de las materias primas, el desarrollo $y$ el financiamiento internacional, en la Conferencia de París sobre Cooperación y Desarrollo. La solidaridad deI Tercer Mundo no se ha resquebrajado descle entonces, no obstante que includablemente el aumento de los precios del petróleo ha golpcado más severamente a los países en clesanollo no exportadores de este producto que a los paises industrializados. Gracias a esta solidariclacl, la problemática Norte-Sur ha tenido una fuorte presencia o incluso

GRichard H. Ullman, "Trilateralism: Partnership for What?", en Foreign Affairs, octubra 1976 .

"Stephen Kasner, "Oil is the Fxception", en Foreign Policy, No 14, 1974. 
hat clominado las discusiones manteniclas en diversos foros internacionalcs -no solamente en aquellos especialmente establecidos para el examen de estas relaciones, como la UNCTAD o el Diálogo cle Paris, sino Lambién clel Consejo Mundial cle Alimentos, la Conferencia Mundial sobre Empleo de la Organización Internacional del Trabajo, la Conferencia sobre el Derecho del Mar y las últimas reuniones del Banco Mundial y del Fondo Monetario Internacional. Lo que es más importante aunque con frecuencia se olvida, es que ello no obedece a una posición reciente sino a una estrategia elaborada en forma colectiva desde hace largos años, ya sea en las Conferencials de Paises no Alineados iniciadas en Bandung en 1955 - que a partir de 1970 pasaron a tener un contenido predominantemente económico- o a partir de los años 1960, a través clel grupo de lọs 77, dentro del marco de la UNCTAD. Resutla claro que esta solidaridad no se basa tanto en la percepción de los beneficios inmediatos que los países del Tercer. Mundo puedan lograr a través de su participación manconunada en las negociaciones internacionales, ni de la mayor o menor equidad con que estos beneficios se distribuyan entre los diversos tipos de países que integran este boque, sino en la percepción más general de que las reglas del juego establecidas por laś grandes potencias durante el periodo colonial operan sistemáticamente en contra de sus intoreses, $y$ de que para modificarlas se requiere una acción concertada, de una manera similar a la forma en que los trabajadores de los Estados Unidos se unieron en el decenio de 1930 para obtener un "nuevo trato".

A esta percepción politica se une la de las oportunidades que el actual contexto internacional ofrece en el plano económico, y la de la creciente importancia que tiene el Tercer Mundo para los paises industrializados, como lo indica cl hecho de que los paises en desarrollo no exportadores cle petróleo representan para los Estados Ưinidos un mercado de 27 mil millones de dólares, més importante que el de los países de la Comunidad Económica Europea, Europá Oriental y la Unión Soviética en conjunto, y por lo tanto una fuente de dos millones de empleos adicionales en los próximos I0 años-de mantenerse las tendencias actuales - así como una fuente de exportaciones de biencs de consumo que podrian contribuir eficazmente a la lucha contra la inflación y de materias primas esenciales pará la industria norteamericana.

Istas realidades han dado impetu y colcerencia a lá plataforma elaboracla por el Tercer Mundo para avanzar hacia el cstablecimiento de un nuevo orden económico internacional. Importa ahora examinar hasta qué punto esta plataforma se ha ido elaborando en una dirección correcta. 
NECESIDAD DE UNA REVISIÓN CRITTICA

DEI. NUEVO ORDEN ECÓNOMICO INTERNAGIONAT.

Los países del Tercer Mundo han logrado anticular un conjunto de ideas muy importantes para el establecimiento de un Nucvo Orrlen Económico Internacional, y las han expresado a tavés de la Resolución y el Programa de Acción aprobados al respecto por la Asamblea Ceneral de las Naciones Unidas, o han contribuido a reficjarlas en un conjunto de Estudios e informes de gran valor -ia mayor parte cle los cuales han sido claborados en los paises inclustrializaclos-entre los más recientes de los cuales se cuenta el informe RIO dirigido por el profesor Jan Timberger. 8

Influidos tal vez por estos éxitos logrados en el plano conceptual, los países del Tercer Mundo han tendido a consiclerar el conjunto de ideas y proposiciones anteriormente mencionado como un programa de acción efectivo y, en mayor o menor medida, se han comprometicio a trabajalr por su implementación a través cle una varicdad cle foros y acciones en el plano internacional. La Conferencia de París y la UNCTAD IV han sido los marcos más relevantes adoptados por estos países para impulsar la implementación de las icieas y medidas que conforman el NOEI. Los escasos resultados que se han logrado en Paris y el camino sin salida a que cn la práctica se ha llegado, después de la reunión cle Nairobi, en relación con la iniciativa Ilamada a constituir la "viga macstra" del nuevo orden -el Prograna Integrado de Productos Básicos- ponen de manifiesto algo que no debió pasar inadverticlo: que el NOEI jamás llegó a constituir un programa de acción efectivo.

Los paises del Tercer Mundo han incurrido en cl defecto lógico de atribuir una "concresión fuera cle lugar" al NOEI, al asignarle el carácter de un programa de acción monolítico, por razones preponderantemente politicas, pero con dudosos resultados económicos. De acuerclo con nuestra percepcion, el NOEI no constituye un programa, sino a lo sumo una agenda para ordenar la discusión que se está desarrollando en torno al reordenamiento de las relaciones NorteSur $-y$ tal vez ni siquiera una agenda, sino el planteamiento de una problemática. Láa calasa principal de esta confusión, que constituye

s Reshaping the International Order, 1976. Ver tambien en informe tituladu What Now? Another Development, de la Fuadación Dag Hammarskjöld, 1975; el Symposism on The New International Economic Order, publicado en Lis Hay'a en 1975, y más recientemente el estudio encomendado al profesor Harlan Cleveland por el Aspen Institutc, titulado 'The 'Third 'Ty' at World Order, 1977. 
la primera cle las falacias a que hacíamos referencia más arriba, radica en que nunca se explicitaron suficientemente lis profundas $-y$ tal vez irreconciliables, de no mediar hábiles esfuerzos para alcanzar ciertos compromisos- diferencias existentes entre la filosofía "libre. cambista" a que los países industrializados permanecieron adheridos $\rightarrow$ al menos en forma maniliesta- en sus esfuerzos por mantener el antiguo orden económico y la filosofía "intervencionista" adoptada por la mayor parte de los paises en desarrollo en sus propuestas relativas a la problemática Norte-Sur, orientadas a lograr una redistribución global clel ingreso mediante un alto givado de regulación de los mercados, obteniclo mediante la aplicación de un conjunto de medidas cle carricter general.

En la generalidad de esas mediclas radica la segunda falacia a que se hacia mención, a saber, la de que problemas de alcance global como los que actualmente enfrentia la comunidad intemacional deben ser resueltos necesariamente -0 , al menos, con ventajas- a través de medidas de carácter jgualmente general. Esta preferencin es notoria en la mayor parte de los informes anteriormente mencionados como, por ejemplo, el Informe RIO, que propone en el largo plazo la creación de una Tesorería Mundial basacla en un "impuesto internacional" para resolver los problemas monctarios y financieros; el fortalecimiento del Fondo para el Desarrotlo a Largo PIazo de la Agricultura en el Tercer Mundo entre las principales medidas para incrementar la producción mundial de alimentos; la adopción de una "estrategia intemacional de industrialización", y un programa automático encaminado a reducir los márgenes de protección pari passu con el nivel de clesarrollo de los paises, a fin de cumplir hacia el año 2.000 con los objetivos de la Conferencia de Lima en el sentido de transferir el $25 \%$ de la producción inclustrial a 105 palses del Tercer Mundo; el establecimiento de una Agencia Mundial para los Recursos Minerales; la creación de una Autoridad Mundial para el Desarrollo Tecnológico, y otras medidas globales para la solución de los problemas del desarrolio internacional, prestando escasa atención a la identificación de vias altemativas para resolver problemas globales mediante un conjunto de acciones parciales y diferenciadas, dependiendo de los sectores y paises involucrados.

La tercera falacia consiste en In tendencia a visualizar a los paises del Tercer Nunclo como un conjunto lomogéneo, cuy'a identidad de intereses los llevará tarde o temprano a adoptar una plataforma común, no sólo en lo que se reliere a su orientación general, sino también en el plano de las soluciones concretas. No se han percibido en toda su extensión las oportuniclades y clesalfos que los paises del Tercer Mundo enfrentan al alcanzar etapas intermedias de des- 
arrollo frente a un mundo industrializado expuesto a serias presiones que deberían inducirlo a hacer pleno uso de la teoria de las ventajas comparativas que por tanto tiempo han preconizado, y a beneficiarse de una división más racional del trabajo a escala intexnacional. Tampoco se han percibiclo plenamente las posibilidades que se presentan dentro de un contexto de escasez, en que a la dependencia económica y tecnológica de los paises en desarrollo frente a los países industrializados viene a agregarse la dependencia estratégica de estos últimos con respecto a los recursos naturales de que disponen los primeros. Estas dos últimas consideraciones llevan a la conclusión de que los páses del Tercer Mundo habrán de ensayar caminos de desarrollo cada vez más coherentes con sus ventajas comparativas y su dotación de factores -caminos que, por consiguiente, serán cada vez més diferenciados.

La superación de las falacias anteriormente mencionadas podría abrir el camino para la concertación de arreglos de mediano y largo plazo, entre los países del Norte y las naciones del Sur, para lograr una distribución de las actividades económicas basada en una mejor división internacional del trabajo, para mutuo beneficio, sector por sector.

La concertación de estos arreglos supone un conocimiento profundo $y$ una aguda percepción cle las necesidades y complementasiedades existentes entre ambos grupos de paises -asi como entre paises determinados en el interior de cada uno de estos bloquesa fin de poder identificar aquellos sectores más propicios para poder desarrollar relaciones de interclependencia, primeramente en el seno de determinadas agrupaciones de países, y finalmente a escala mundial

Este enfoque, por otra parte, podría estimular la tendencia hacia una mayor confianza colectiva en sí mismos y una mayor ayuda mutua entre los paises en desarrolio. En efecto, el establecimiento de relaciones de interdependencia basadas en la identificación cle intereses comunes, no sólo supone operar durante convenios de mediano y largo plazo sector por sector, sino que también exige una mayor articulación entre aquellas naciones pertenecientes a cada uno de ambos bloques, vinculadas por determinados intereses específicos.

Lo anterior supondria que el modesto pero históricamente significativo incremento que ha experimentado el poder de negociación de los países del Tercer Munclo durante los últimos años debería ser aplicado, precisamente, a iclentificar oportunidades de cooperación con los paises industrializados, en cuya búsqueda la negociación debe reemplazar a la confrontación.

Naturalmente, para ello es necesario que los paises capitalistas del 
Norte aprendan a reconocer los intereses de los paises del Sur, y a aceptar arreglos más equitativos que en el pasado.

Un ejemplo del tipo de proposiciones a que poclria dar lugar el "diálogo de sordos" que se produce cuando no existe esta disposición por parte de ambos bloques, se refiere a la contraposición entre las propuestas formuladas por los paises del Tercer Mundo en relación con el Programa Integrado de Productos Básicos, por una parte, y a las que efectuaran los Estados Unidos en el seno de la UNCTAD IV respecto al establecimiento de un Banco Internacionad en Santiago de Chile, en ocasión de la penúltima Asamblea General de la Organización de Estados Americanos, dentro del ámbito hemisférico.

América Latina, concebida como un ámbito geográfico en que coexisten países en desarrollo con paises semiindustrializados, constituye un campo de experimentación propicio para avanzar hacia el establecimiento de un sistema de relaciones políticas y económicas entre los países inclustrializados y los países en desarrollo, cimentadas en la identificación de intereses reciprocos, en que la confrontación sea paso a la interdependencia.

\section{IA PARTICIPACIÓN INTERNACIONAL DE AMÉRICA LATINA EN LA DÉCADA DE 1970}

En electo, los cambios que han tenido lugar en la economía internacional clurante los últimos años, a que se hacía referencia más arriba, han tenido su correla to en la evolución económica de América Latina.

De acuerdo con el pensamiento prevaleciente en el desarrollo de 1950, las economias latinoamericanas habrian de continuar especializándose en el comercio de productos básicos y seguirían viéndose afectadas por el deterioro de los términos de intercambio entre las materias primas que constituian ei grueso de sus exportaciones y los productos industriales que debcrían importar para satisfacer la demanda de bienes de consumo y de capital, generada por su propio proceso de desenvolvimiento económico. Como consecuencia de lo anterior, America Latina contaria con escasas posibilidades de exportar productos manufacturados y experimentaría crecientes dificultades para hacer frente a sus necesidades de importaciones esenciales, lo cual la encauzaria por el camino de la industrialización sustitutiva, y' la llevaría a desarticularse en forma gradual e inexorable del contexto internacional.

Este modelo involucraba determinadas predicciones respecto del 
comportamiento de la economia mundial y de las politicas de desarrollo de los paises latinoamericanos. Entre las primeras se contaban el mantenimiento o la acentuación de las tendencias prevalccientes por aquel entonces en cuanto a una marcada cstratificación del sistema internacional, basada en el papel hegemónico de los Estaclos Unidos en la cconomia mundial; el creciente estrechamiento del margen de que dispondrian los paises en desarrollo para diversificar sus relaciones económicas externas, $y$ el continuo clcterioro de la posición de los procluctos bísicos en el comercio internacional, debiclo a su vulnerabilidad intrinseca. Entre las segundas se señalaban, fundamentilmente, la adhesión de los gobiernos latinoamericanos a un modelo de desarrollo relativamente protegido, basado en la inclustrialización sustitutiva y orientaclo hacia la ampliación del mercado interno mediante un conjunto de medidas moderadamente redistributivas, dentro de una básica similaridad de sus sistemas políticos. Los cambios octrridos en los últimos años en los planos nacional e internacional han tendiclo a considerar la valiclez de cstos supuestos.

En efecto, como ya se ha señalado, durante el decenio de 1970 se advierte una notable profundización de las relaciones de interdependencia entre todas las naciones del mundo.

Ello se traduce, por una parte, en una tendencia hacia la revalorización de los recursos naturales en la economía mundial. No es extaño, por consiguiente, que los paises latinoamericanos adopten politicas y medidas encaminadas a adquirir un. mayor grado de control y de procesamiento local de sus recursos naturales, y manifiesten el deseo de reencauzar su proceso de industrialización con el objeto de basarlo cada vez más en la utilización de sus recursos propios."

Al mismo tiempo, América Latina está clejando de producir exclusivamente bienes primarios. Aunque los produclos básicos todavia representan la mayor parte de sus ventas al exterior, sus exportaciones de manufacturas se han expandido y diversilicacio en forma no imaginable hace 10 ó 15 años, y el desenvolvimiento industrial y tecnológico de algunos paises que estai alcanzando niveles de cierta relevancia en determinados sectores. Durante estc último periodo, las políticas económicas de algunos paises de la región han cstado adaptúndose a los requerimientos propios de economias mis abiertas y' competitivas, han reducido Jos márgenes de protección prexistentes o los han tornado más selectivos, y han desarrollado un conjunto de instrumentos para acceder a los mercados externos con productos no

$9 \mathrm{Ver}$, del autor, el trabajo Tendencias favorables o Actrersas a ha formación de un Subsistema Regional Latinonmericano, en Estudios Internacionales No 20, enero de 1975, y Elementes para un estudio sobre los procesos de Integración $y$ de Cooperación en América Latima, en Comercio Exterior, febrem de 1977, jp. 190 y 191 . 
tradicionales. A su vez, el rolumen y la composición de sus imṕortaciones cambiaba sustancialmente, y América Latina se trans[ormaba en: un comprador importante para muchos paises industrializados, lo'cual generaba, por una parte, un poder de negociación insuficientemente utilizado $y$, por la otra, un potencial de sustitución de importaciones en una escala diferente a las experiencias del pasado-así como de expansión del comercio intrarregional que incluso pudiera superar los logros ya alcanzados dentro de los esquemas tradicionales de integración económica.

Al mismo tiempo, el origen y composición del financiamiento externo ha variado en forma sustancial, aumentando drísticamente el monto de la deuda extema y disminuyendo la participación relativa de las fuentes oficiales, conforme algunos países de la región incrementaban sus oportunidacles de acceso a los mercados privados de capital.

Para hacer posibles estos cambios, con frecuencia fue necesario sacrificar las metas más igualitarias incorporadas en las políticas refor* mistas de comienzo de los años 1960, lo que en muchos casos dio lugar a la ruptura del consenso social y a la entronización de regimenes autoritarios, con la consiguiente acumulación de tensiones sociales que pugnan por reencauzar el proceso dentro de marcos más justos y más participatorios.

No obstante los costos sociales involucrados en las tenclencias anteriorinente señaladas, es includable que la forma de inserción de Amé. rica Latina en la economía mundial estí experimentando una transformación muy profunda, transformación que habrá de ser asumida por el nuevo consenso social que emerja como consecuencia de las presiones mencionadas.

Todo ello hace necesario replantear las formas que deberia adoptar la participación de América Latina en el sistema internacional tomanclo en cuenta, por una parte, la mayor capacidad de respuesta y de participación en el sistema que ha revelado lit región durante los últimos años $y$, por la otra, la necesidad de remover los graves problemas que aún afectan su relacionamiento externo. Lo que es más iniportante, urge repensar el papel de una región semidustrializada, como América Latina, en la nueva división internacional del trabajo que parece estarse gestando en la economia mundial.

No se han analizado con la profundidad que seria de desear los factores que explicarian la capaciclad que han clemostrado los países latinoamericanos para reaccionar frente a los efectos combinados de la recesión experimentada por las economías centrales y el aumento de los precios de los combustibles y los bienes inclustriales que se ven en la necesiclad de importar para asegurar la continuidad de su pro- 
ceso de desarrollo. Lintre estos factores podrian contarse una mayor diversificación de las cconomías y las exportaciones de los paises latinoamericanos; su mayor capacidad para producir internamente los insumos $y$ bienes de capital que anteriormente importaban; sus mayores posibilidades cle acceso al financiamiento internacional, especialmente de origen privado; su mayor desarrollo tecnológico, y una activa participación del Estado en cicrtos sectores claves del clesarrollo nacional que en el pasado habrian quediclo casi exclusivamente en manos de las empresals transnacionales.

No obstante lo anterior, también hace falta explicar la subsistencia de viejos problemas y el surgimiento de nuevos interrogantes, que continúan dificultando el relacionamiento exterior de América Latina. No se ha insistido suficientemente en lat paradoja de que una región que tradicionalmente ha sido exportaclora cle productos primarios no haya seguido el incremento de la demanda mundial de materias primas y alimentos -que aumentó en poco más cle un $4 \%$ anual entre 1960 y 1970 contra un incremento del $3,8 \%$ por año de las exportaciones primarias de los países Iatinoamericanos $\rightarrow$ ya sea como consecuencia de las conocidas condiciones adversas de los mercados internacionales de productos búsicos o de la escasa prioridad otorgada a clichos sectores por las politicas económicas cle los paises latinoamericanos durante el período mencionado. Tampoco se ha puesto de manifiesto, en la extensäón que sería de desear, la pradoja de que países como los Estados Uniclos, que han defendido el libre comercio y la teoría de las ventajas comparativas, la vulneren constantemente en desmedro de los países en desarrollo abrazando un nuevo proteccionismo que se traduce, por ejemplo, en el hecho de que los 106 productos latinoamericanos expuestos a restricciones cuantitativas debido a su carácter competitivo hayan estaklo sujetos en 1975 a un arancel medio del $9 \%$, en comparación con el promedio de los Estados Unidos, que en ese mismo año fue sólo del $5,8 \%$. No existe consenso en tomo a los beneficios y a los peligros inherentes al crecimiento de la deuda cxterna, que en lo que vá corrido del clecenio aumentó cle 35 a cerca de 70 mil millones de clólares, originándose los clos tercios de los nuevos endeudamientos en los mercados privados de capital, ya que este mayor endeudamiento ha sido utilizado al parecer por los paises de la región como una alternativa a la recomendiación convencional cte buscat el equilibrio externo a traves de politicas recesivas, y porque los estaclos que ostentan un grado de endeudamiento mayor son precisamente los que parecen menos preccupados al respecto. Reina también ambigäiedad acerca de las consecuencias que podría acarrear para esos países la "privatización" de la cleuda, ya que si bien por una parte esta modalical de 
financiamiento ofrece ventajas en cuanto al la tapiciez cle las negociaciones y la falta de condicionamientos en el otorgamiento de los préstamos, existe un riesgo cumulativo de que el renegociamiento de estos créditos (generalmente de corto plazo) se sujete a la adopción, por parte de los deudores, de recomendaciones ortodoxits formuladas por el Banco y el Fondo, como consecuencia de las presiones que el Fecleral Reserve ya ha comenzaclo a ejercer sobre la banca privada de los países de la región. Aun no se han sacalo todas las consecuencias del hecho de que hacia 1975 las ventas de fas filiales de empresas transnacionales de América Latina hayan alcanzado a alrededor de 80 mil millones cic dólares, esto es, alrecledor de la cuarta parte clel valor de la producción corresponcliente a los sectores primario y secundario - concentrándose, precisamente, en las activiclades más clinúmicas.

La inclustrialización basada en la sustitución cle importuciones dio lugar tambićn a la paradoja de que, sienclo los países latinoamericanos tradicionalmente agrícolas y' estando dotados de vastos recursos naturales, hayan clcjado hasta ahora casi enteramente en manos extranjeras la transfornación de esos recursos e importen cuantiosos volúmenes de alimentos y materias primas minerales. El procesamiento local de esos productos no sólo es condición esencial de la soberanía de los paises de la región sino también una base indispensable para completar su estructura industrial, generar mayor cantidad de empleos y aumentar el valor agregado de sus exportaciones. Un desarrollo basado en los recursos humanos, tecnológicos y naturales y en las ventajas comparativals que posee la región podría constituir el mejor camino para que ésta obtenga mayores beneficios dentro de in nueva división internacional clel trabajo que se anticipa dentro de un mundo cada vez mis interdependiente.

También habría que replantear los conceptos que inspiraron los primeros movimientos de integración económica entre los paises de la región. Como ya se ha señalado, el modelo de clesarrollo prevaleciente en el decenio de 1950 predecía una continua especialización de los paises Jatinoamericanos en el comercio de productos búsicos y una creciente caída de su capacidad para importar, todo lo cual implicaba una paulatina desarticulación respecto del contexto munclial. Con el objeto de comprimir el coeficiente de importaciones y de estimular el comercio de manufacturas, se preconizaban politicas de industrialización basadas en la sustitución de importaciones. Estos procesos estaban llamados a encontrar limites relativamente próximos dentro de las estrechas climensiones de los mercados nacionales, limites que debian superarse mediante la formación de "mercaclos comunes" regionales relativamente protegidos, concepciones que clieron lugar al esta- 
blecimiento cic la ALALC y el MCCA y posteriormente, al Acuerdo de Cartagena.

La crisis o estancamiento que en mayor o menor medida están experimentanclo los esquemas globales de integración han iclo acompañados por un marcado dinamismo en lo que se reficre a la promoción de otras formas de cooperación entre dos o más paiscs del área. Estas nucvas modalidades se expresan en un conjunto de acuerdos o proyectos de complementación económica, a través de los cuales los paises de la región buscan poner en común mercados, recursos o tecnología, no tanto con el objeto de atender la demanda interna e incrementar el comercio intrarregional, sino con el de atumentar su eficiencia y competitividad en los mercados internacionales.

Toclo ello tiende a poner en tela de juicio los supuestos básicos de la teoria convencional de la integración $y^{*}$ a plantearse el interrogante acerca del papel que está llamada a desempeñar la complementación económica entre los paises del área - particulamente entre los pitises mayores y los más pequeños- en sus esfuerzos encaminaclos a lograr una mejor participación en la división internacional del trabajo.

LAS RELACIONES NORTE-SUR: ALTERNATIVAS PARA EL FUTURO

Las principales corrientes de pensamiento en. que se ha polarizado el debate Norte-Sur, a que se hacia referencia en las secciones precedentes, no pirecen corresponder a las oportunidades y requerimientos de un mundo caracterizaclo por la consolidación de estrechas relaciones de interclependencia.

La filosolia "librecambista" proponc como objetivo la maximización de lat eficiencia y clel crecimiento cconómico, tanto a nivel nacional como internacional, consicicranclo que el crecimiento es mutuamente beneficioso para todos los estados aún cuando la distribución de sus beneficios no resultare equitativa. Este objetivo puede lograrse mediante una óptima asignación de los recursos disponibles a tavés de las señates provenientes clel mercado dentro de un sistema regido por el liberalismo cconómico. Lo anterior implica que los paises abran sus economias al capital extranjero y a los bienes importados y se especialicen en la proclucción de aquellos bienes para los cuales poseen ventajas comparativas. De esta manera se incrementa la producción mundind $y$, a la largat, todos los estados restultan beneficiatos. Dentro cle estas premists, no existe ninguma rałón para prescribir políticas diferentes para las distintas economias, ni pam temer que surjan 
conflictos profundos y persistentes en las relaciones econónicas entre los distintos estados.

De acuerclo con la filosofia "intervencionista", acuñada por los paises del Tercer Mundo durante los últimos decenios, aunque el crecimiento económico y la eficiencin constituyen objetivos prioritarios, la igualdad en la distribución del ingreso y la autocleterminación nacional involucran valores más cruciales. A la luz de esta percepción, las relaciones económicas entre los estados tienen lugar dentro de un marco de reglas del juego definidas por las potencias hegemónicas, que discriminan en contra de los países más débiles, danclo lugar a una injusta distribución de los beneficios derivados del proceso. La apertura de las economías de los países más débiles y su especialización en la producción de determinados bienes sólo tendría el efecto de permitir a los más poderosos ganar la competencia por el acceso a los recursos y mercados mundiales, acentuando la desigual distribución de esos beneficios, y creando profundos conflictos entre ambas categorías de países. Obviamente, desde este ángulo, no es posible prescribir el mismo tipo de política para ambos grupos de paises.

Mrientras que para el primer enfoque las reglas del juego internacional son básicamente correctas, y el desarrollo depende fundamentalmente de la idopción de politicas adecuadas por parte de los paises del Tercer Mundo, para el segundo la falla está incorporada en la estructura del sistema internacional, que discrimina en contra de los países más débiles, los cuales requieren por consiguiente cle un tratamiento prefcrencial que sólo puede lograrse a través de un grado considerable de regulación de los mercaclos.

El clemento que falta de los análisis precedentes es el reconocimiento del proteccionismo que parece estal ganando terreno en las relaciones internacionales. Un número cacla vez mityor de paises, en el mundo industrializado, están buscando poner controles a las importaciones en orden a proteger su industria cloméstica y a impedir el aumento del desempleo. Los paises ricos se acusan recíprocamente de emplear prácticas comerciales desleales, micntras que a su vez son acusados de cerrar sus mercados a las manufacturas de los paises subdesarrollados. Unos y otros compiten por el acceso o el control cle las fuentes de materias primas. Las corporaciones multinacionales, que una vez ponian en jique la soberania nacional, hoy estín siendo jaqueadas tanto en los países en desarrollo como en las naciones industrializadas, deseosas de mantener sus niveles de activiclad económica y' sus fuentes de empleo. 10

Estas consideraciones podrian inducir a ambos grupos de paises a

10 Vêase de Raymond Vernon, autor de Sovereignty at Bay, "Storm Over the Multinationals: Problems and Prospects", en Foreign Affairs, enero de 1977. 
buscar arreglos sectoriales de largo plazo, como se sugeria en un comienzo, en relación con la distribución de determinadas producciones o actividades económicas y con el logro de una mejor división. internacional del trabajo, que contemplen adecuadamente sus intereses reciprocos,

En este terreno ignoto, planteado por las tendencias hacia un neomercantilismo anteriomente esbozadas, uno podria aventurarse a formular algunas hipótesis de trabajo, extremadamente tentativas, acerca de las modalidades que pudieran adoptar las relaciones internacionales dentro del contexto señalado.

Estas hipótesis se inspiran en una analogía histórica, a saber; la evo. lución de las relaciones sociales del mundo occidental -que en aquella época equivalían a las relaciones intemacionales entre los modernos estados- con ocasión de Iu caida del Imperio Romano. Frente a este desafio histórico, se ensayaron tres tipos de respuesta, representadas por el modelo "liberal", que hubiese sido asegurado nediante la supervivencia del imperio; por el modelo "centralizado", encarnado en la experiencia bizantina, y por el modelo feudal, bajo el cual se organizó efectivamente la sociedad medieval.

Este último representó un compromiso encaminado a facilitar lat reconstrucción de las relaciones sociales preexistentes durante el imperio, mediante una estrategia sectorial o "por piezas", después de la ruptura experimentada por un orden imperial pretendidamente ecuménico, pero en efecto provinciano, por obra de la irrupción ciel "proletariado externo" descrito por Toynbee.

Del mismo modo, pareceria que el sistema "librecambista" o neoliberal que, con igual pretensiones de validez universal, emergió de Bretton Woods, está siendo desbordado, como consecuencia de la emergencia de un proletariado externo, representado por los países emergentes del Tercer Mundo, cuya actitud contestataria se basa en su percepción de haber sido incorporados dentro de un orden cuyas reglas no contribuyeron a establecer.

Dentro de esta confrontación, Jos países inclustrializaclos han insistido en el mantenimiento del orden neoliberal, mientras que los páses en desarrollo han abrazado un programa encaminado a obtener un elevado grado de regulación de ese sistema. Alternativamente, si dicho grado de regulación fuese inaceptable para los países industrializados y/o inconveniente para los paises en desarrollo, podría llegarse a unit reestructuración del orden mundial de corte "neomercantilista", basada en agrupaciones de productos y de actividades económicas, y/o en agrupaciones de paises. Como se ha anticipado, ello podria involucrar arreglos de largo plazo entre los paises industrialirados y los paises en desarrollo, que contemplaran los intereses de estos últimos 
en mejorar sus términos de intercanbio con los países centrales y dé obtencr una mejor participación en la división internacional del trabajo, asi como los intereses de los primeros en el sentido de asegurar su abastecimiento de energia y de materias primas. Este camino podría involuctar el riesgo de una mayor fragmentación de las relaciones internacionales $-\mathrm{c}$ incluso sle una incleseable verticalización cle las mismas- pero no puede negarse que, al fin y al cabo, representa una de las formas posibles de poner término al presente desorden intemacional, que merece mayor atención que la que se le hat prestado hasta ahora. 